\title{
Phage on Tap - A quick and efficient protocol for the preparation of bacteriophage laboratory stocks
}

\author{
Natasha Bonilla ${ }^{1}$ ， Maria Isabel Rojas ${ }^{1}$, Giuliano Netto Flores Cruz ${ }^{1}$, Shr-Hau Hung ${ }^{1}$, Forest Rohwer ${ }^{1}$, \\ Jeremy J Barr Corresp. 1 \\ ${ }^{1}$ Department of Biology, San Diego State University, San Diego, CA, United States \\ Corresponding Author: Jeremy J Barr \\ Email address: jeremybarr85@gmail.com
}

A major limitation with traditional phage preparations is the variability in titer, salts, and bacterial contaminants between successive propagations. Here we introduce the Phage On Tap (PoT) protocol for the quick and efficient preparation of homogenous bacteriophage (phage) stocks. This method produces homogenous, laboratory-scale, high titer (up to $10^{10-11} \mathrm{PFU} \cdot \mathrm{ml}^{-1}$ ), endotoxin reduced phage banks that can be used to eliminate the variability between phage propagations and improve the molecular characterizations of phage. The method consists of five major parts, including phage propagation, phage clean up by $0.22 \mu \mathrm{m}$ filtering and chloroform treatment, phage concentration by ultrafiltration, endotoxin removal, and the preparation and storage of phage banks for continuous laboratory use. From a starting liquid lysate of $>100 \mathrm{~mL}$, the PoT protocol generated a clean, homogenous, laboratory phage bank with a phage recovery efficiency of $85 \%$ within just two days. In contrast, the traditional method took upwards of five days to produce a high titer, but lower volume phage stock with a recovery efficiency of only $4 \%$. Phage banks can be further purified for the removal of bacterial endotoxins, reducing endotoxin concentrations by over 3,000-fold while maintaining phage titer. The PoT protocol focused on T-like phages, but is broadly applicable to a variety of phages that can be propagated to sufficient titer, producing homogenous, high titer phage banks that are applicable for molecular and cellular assays. 
5 Natasha Bonilla ${ }^{1}$, Maria Isabel Rojas ${ }^{1}$, Giuliano Netto Flores Cruz ${ }^{1}$, Shr-Hau Hung ${ }^{1}$, Forest

6 Rohwer $^{1}$, and Jeremy J. Barr ${ }^{*}$

7

8 'Department of Biology, Department of Biology, San Diego State University, 5500 Campanile Dr, San Diego, CA

9 92182-4614, USA

10

11 *To whom correspondence should be addressed. Email: jeremybarr85@gmail.com

12 


\section{Abstract}

14 A major limitation with traditional phage preparations is the variability in titer, salts, and 15 bacterial contaminants between successive propagations. Here we introduce the Phage On Tap 16 (PoT) protocol for the quick and efficient preparation of homogenous bacteriophage (phage) 17 stocks. This method produces homogenous, laboratory-scale, high titer (up to $10^{10-11} \mathrm{PFU} \cdot \mathrm{ml}^{-1}$ ), 18 endotoxin reduced phage banks that can be used to eliminate the variability between phage 19 propagations and improve the molecular characterizations of phage. The method consists of five 20 major parts, including phage propagation, phage clean up by $0.22 \mu \mathrm{m}$ filtering and chloroform 21 treatment, phage concentration by ultrafiltration, endotoxin removal, and the preparation and 22 storage of phage banks for continuous laboratory use. From a starting liquid lysate of $>100 \mathrm{~mL}$, 23 the PoT protocol generated a cleaned, homogenous, laboratory phage bank with a phage 24 recovery efficiency of $85 \%$ within just two days. In contrast, the traditional method took upwards 25 of five days to produce a high titer, but lower volume phage stock with a recovery efficiency of 26 only 4\%. Phage banks can be further purified for the removal of bacterial endotoxins, reducing 27 endotoxin concentrations by over 3,000-fold while maintaining phage titer. The PoT protocol 28 focused on T-like phages, but is broadly applicable to a variety of phages that can be propagated 29 to sufficient titer, producing homogenous, high titer phage banks that are applicable for 30 molecular and cellular assays. 


\section{Introduction}

34 Due to increasing interest for the use of bacteriophage (phage) in medical, industrial, and 35 molecular settings, new approaches are required to quickly and efficiently produce high titer, 36 homogenous, and purified phage stocks. It is desirable that these stocks be free of bacteria, 37 molds, debris, culture medium, and bacterial endotoxins (Adams, 1959). Typically the ability to 38 produce high titer phage stocks is largely dependent on the particular phage and host cell under consideration, yet certain principles and methodologies can be broadly applied. Traditional

40 techniques used for the concentration and purification of phage involve centrifugation, filtration, 41 ultrafiltration, precipitation with Polyethylene Glycol (PEG), ultracentrifugation in cesium 42 chloride $(\mathrm{CsCl})$ gradients, and dialysis (Adams, 1959; Yamamoto et al., 1970; Seeley \& 43 Primrose, 1982; Suttle, Chan \& Cottrell, 1991; Carlson, 2004; Bourdin et al., 2014). Yet many of 44 these techniques are time consuming and affect phage recovery and/or viability.

46 Phage preparations are often contaminated by macromolecules derived from the host bacteria 47 and culture media, with the major pyrogen being the lipid A moiety of lipopolysaccharide 48 (endotoxin) from the outer membrane of Gram-negative bacteria (Raetz et al., 2007). Endotoxins 49 are amphipathic molecules; the lipid component is linked to a core polysaccharide and as a result 50 they can form large aggregates greater than $1000 \mathrm{kDa}$ in solution (Batista et al., 2013). 51 Endotoxin elicits a wide variety of pathophysiological effects in the body. Exposure to even 52 small amounts can result in toxic shock, cell injury, cytokine production and the activation of 53 immune responses (Morrison \& Ulevitch, 1978; Rietschel et al., 1994; Alexander \& Rietschel, 54 2001; Pabst et al., 2008). Due to these effects it is important that endotoxins be removed from 55 phage preparations when studying or applying phage in the context of eukaryotic systems. The 56 amount of endotoxin is defined as an endotoxin unit (EU), which corresponds to the activity of $57100 \mathrm{pg}$ of E. coli lipopolysaccharide. The endotoxin content of distilled water is estimated at 20 $58 \mathrm{EU} \cdot \mathrm{ml}^{-1}$, with the allowed limit for intravenous and oral administration set at $5 \mathrm{EU} \cdot \mathrm{kg} \cdot \mathrm{hr}^{-1}$ and $59<20 \mathrm{EU} \cdot \mathrm{ml}^{-1}$ respectively (Bruttin \& Brüssow, 2005; Gorbet \& Sefton, 2005; Abedon et al., 60 2011).

61

62 A further limitation with traditional phage preparations is the variability of titer, salts, and 63 bacterial contaminants produced between successive propagations. Eliminating this variability 
64 has proven critical for accurate analysis for the molecular interactions of phage within the 65 context of eukaryotic hosts (Barr et al., 2013, 2015). Here we present the Phage On Tap (PoT) 66 protocol as a fast and efficient way to produce homogenous laboratory phage stocks. Phage 67 stocks were sterilized by centrifugation, $0.22 \mu \mathrm{m}$ filtration, and chloroform treatment, before 68 concentration and washing using ultrafiltration, and storage at $4{ }^{\circ} \mathrm{C}$ for four months with minimal 69 degradation. However, the Phage On Tap protocol was not able to effectively reduce bacterial 70 endotoxins, likely due to our ultrafiltration-based approach and the large aggregate size of 71 endotoxins. Numerous endotoxin removal procedures and commercial kits are available 72 (Boratyński et al., 2004; Merabishvili et al., 2009; Oślizło et al., 2011; Batista et al., 2013; 73 Branston, Wright \& Keshavarz-Moore, 2015), yet many of these methodologies lack generality, 74 are time consuming, or are cost-prohibitive. Recently, the successful reduction of endotoxins $75\left(<20 \mathrm{EU} \cdot \mathrm{ml}^{-1}\right)$ from phage lysates was reported by extraction with organic solvents (Szermer76 Olearnik \& Boratyński, 2015). We corroborate this organic solvent-based method for the 77 removal of bacterial endotoxin from phage lysates and adapt this methodology, with reduced 78 processing time through the use of speed vacuum, in the Phage On Tap protocol. The PoT 79 protocol can purify phage lysates, with volumes ranging between 50-300 $\mathrm{ml}$ (or greater if 80 required), producing homogeneous phage stocks for further laboratory testing. The method takes 81 two days to purify and concentrate a final phage lysate of $\sim 10 \mathrm{ml}$ of with a titer $>10^{10} \mathrm{PFU} \cdot \mathrm{ml}^{-1}$, 82 and a significant reduction in bacterial endotoxin levels. Our method has been tested on a variety 83 of phages, including T4 (E. coli), T3 (E. coli), T5 (E. coli), and Spp1 (B. subtilis), and is broadly 84 applicable to other tailed phages. 
86 Materials and Methods

87 Reagents:

88 -Luria-Bertani (LB) broth (Fisher Scientific, cat. no. DF0446)

89 -Agar (Fisher Scientific, cat. no. BP1423)

90 -Calcium Chloride Dihydrate $\left(\mathrm{CaCl}_{2} \cdot 2 \mathrm{H}_{2} \mathrm{O}\right)$ (Fisher Scientific, cat. no. C69)

91 -Magnesium Chloride Hexahydrate $\left(\mathrm{MgCl}_{2} \cdot 6 \mathrm{H}_{2} \mathrm{O}\right)$ (Fisher Scientific, cat. no. BP214)

92 -Sodium Chloride ( $\mathrm{NaCl}$ ) (Fisher Scientific, cat. no. S671)

93 -Magnesium Sulfate Heptahydrate $\left(\mathrm{MgSO}_{4} \cdot 7 \mathrm{H}_{2} \mathrm{O}\right)$ (Fisher Scientific, cat. no. M63)

94 -Trizma Hydrochloride (Tris HCl pH 7.4) (Sigma-Aldrich, cat. no. 93313)

95 -Chloroform (Fisher Scientific, cat. no. BP1145) CAUTION: Chloroform is toxic and should

96 only be used in a fume hood and with personal safety equipment, such as gloves and goggles.

97 -Pierce ${ }^{\mathrm{TM}}$ LAL Chromogenic Endotoxin Quantitation Kit (Thermo Fisher, cat. no. 88282)

98 Note: Endotoxin Quantitation Kit is optional and is only required for the quantitation of 99 endotoxins in phage lysates.

100 -Ethanol, Absolute $\left(\mathrm{C}_{2} \mathrm{H}_{5} \mathrm{OH}\right)$ (Fisher Scientific, cat. no. BP2818)

101 -Glycerol (Fisher Scientific, cat. no. G31)

102

103 Equipment:

$104-37^{\circ} \mathrm{C}$ incubator with a rocker

105 -Centrifuge with swinging bucket rotor

106 -Microtube centrifuge

107 -Centrifugal vacuum concentrator

108 -Stir plate

109 -Petri dish with disposable lid (Fisher Scientific, cat. no. 09-720)

$110-1.7 \mathrm{ml}$ microcentrifuge tubes (Fisher Scientific, cat. no. 02-681)

111 -Falcon $50 \mathrm{ml}$ conical centrifuge tubes (Fisher Scientific, cat. no. 14-432)

112 -Small glass test tubes $13 \times 100 \mathrm{~mm}$ (Fisher Scientific, cat. no. 14-958)

113 -Serological pipettes (Fisher Scientific, cat. no. 07-200)

$114-0.22 \mu \mathrm{m}$ Sterivex filter units (Millipore, cat. no. SVGP)

115 -Whatman Anotop $0.02 \mu \mathrm{m}$ sterile syringe filters. (Fisher Scientific, cat. no. 09-926-13) 
116 -Amicon ${ }^{\circledR}$ Ultra-15 centrifugal filter units, Ultracel $100 \mathrm{kDa}$ membrane (Millipore, cat no.

117 UFC910008)

$118 \quad$ Note: $100 \mathrm{kDa}$ membrane pore size is equivalent to a spherical particle with a diameter of

$119 \sim 3 \mathrm{~nm}$ and is therefore sufficient for the capture of all known bacteriophages (Erickson, 120 2009).

$121-500 \mathrm{ml}$ PYREX ${ }^{\circledR}$ screw cap storage bottle with plastic seal (Fisher Scientific, cat. no. 13-700-

$122446)$

123 -Spectra-Por ${ }^{\circledR}$ Float-A-Lyzer ${ }^{\circledR}$ G2 Dialysis membrane, $10 \mathrm{~mL}$, MWCO 3.5-5 kDa (Sigma-

124 Aldrich, cat no. Z726273)

125 -Nalgene ${ }^{\mathrm{TM}}$ General Long-Term Storage Cryo Tubes (Fisher Scientific, cat. no. 03-337)

126

127 Reagent Setup:

128 LB broth: $25 \mathrm{~g} \mathrm{LB}$ broth in 1 liter $\mathrm{dH}_{2} \mathrm{O}$

129 LB top agar: $25 \mathrm{~g}$ LB broth, $7.5 \mathrm{~g}$ Agar, in 1 liter $\mathrm{dH}_{2} \mathrm{O}$.

130 LB agar plates: $25 \mathrm{~g}$ LB broth, $15 \mathrm{~g}$ Agar, in 1 liter $\mathrm{dH}_{2} \mathrm{O}$

$131 \mathrm{SM}$ buffer: $5.8 \mathrm{~g} \mathrm{NaCl}, 2.0 \mathrm{~g} \mathrm{MgSO}_{4} .7 \mathrm{H}_{2} \mathrm{O}, 50 \mathrm{ml} 1 \mathrm{M}$ Tris- $\mathrm{HCl} \mathrm{pH} 7.4$, in 1 liter $\mathrm{dH}_{2} \mathrm{O}$.

132 Autoclave, $0.02 \mu \mathrm{m}$ filter-sterilize before use, and store at room temperature.

133 Calcium chloride $\left(\mathrm{CaCl}_{2}\right)$ : Prepare a $1 \mathrm{M}$ stock solution and add a final concentration of $0.001 \mathrm{M}$

134 to desired volume of the LB broth that will be used for the liquid lysate. Autoclave, $0.02 \mu \mathrm{m}$

135 filter-sterilize before use, and store at room temperature.

136 Magnesium chloride $\left(\mathrm{MgCl}_{2}\right)$ : Prepare a $1 \mathrm{M}$ stock solution and add a final concentration of

$1370.001 \mathrm{M}$ to desired volume of the LB broth that will be used for the liquid lysate. Autoclave, 0.02

$138 \mu \mathrm{m}$ filter-sterilize before use, and store at room temperature. 
140 Phage on Tap (PoT) Protocol:

141 Notes:

142 - The procedure for phage propagation is largely specific for each phage and bacterial host.

143 Here we use propagation conditions for T4 phage and Escherichia coli B bacterial host. It

144 is recommended to use appropriated growth and propagation conditions for your choice of

145 phage and host.

146 - Once a sufficiently high titer phage lysate is obtained please proceed to step 3.

147 - It is recommended to only propagate and purify one phage at a time to prevent cross148 contamination.

149

150 1| Phage plaque assay for determination of titer (Adams, 1959).

151 (i) Grow E. coli B bacterial host in LB broth overnight at $37^{\circ} \mathrm{C}$.

152 (ii) Dilute phage stock or isolate in LB broth down to the desired dilution (e.g., for a phage 153 stock of $10^{8} \mathrm{PFU} \cdot \mathrm{ml}^{-1}$ serially dilute down to $10^{-6}$ and $10^{-7}$ to obtain countable plaques).

154 (iii) Heat LB top agar in microwave until completely molten, then allow top agar to cool in a $15556^{\circ} \mathrm{C}$ water bath or until it is warm to the touch.

156 (iv) Add $1 \mathrm{ml}$ of the overnight bacterial host and $1 \mathrm{ml}$ of the phage dilution to a glass test tube and mix.

158

(v) Add $3 \mathrm{ml}$ of molten top agar to the glass test tube.

(vi) Quickly pour molten mixture onto an LB agar plate and tilt the plate to evenly distribute the agar. Let sit undisturbed until the agar has gelled.

(vii) Once plate has gelled ( $\sim 5$ minutes), invert and incubate overnight at $37^{\circ} \mathrm{C}$.

168 2A| Phage isolation and propagation via plate lysate.

(viii) Count phage plaques and determine phage titer in plaque-forming units $\left(\mathrm{PFU} \cdot \mathrm{ml}^{-1}\right)$ using the following formula: $P F U$ per $\mathrm{ml}=$ plaques per plate $\times$ volume plated in $\mathrm{ml} \times$ dilution factor e.g., if there are 20 plaques when you plated out $1 \mathrm{ml}$ from the $10^{6}$ dilution, the titer of the phage stock is $2.0 \times 10^{7} \mathrm{PFU} \cdot \mathrm{ml}^{-1}$.

\footnotetext{
(i) From a plate lysate plate pick a single phage plaque using a sterile Pasteur pipet.
} 
170 (ii) Resuspend the plaque into a microcentrifuge tube containing $1 \mathrm{ml}$ of filter-sterilized 171 phage diluent (SM buffer) and vortex for $5 \mathrm{~min}$.

172 (iii) Centrifuge at $4,000 \mathrm{x}$ g for $5 \mathrm{~min}$ to remove any remaining debris.

173 (iv) Perform plate lysate as described above using the resuspended phage.

174 (v) After incubation the entire plate should be lysed. Pour $\sim 5 \mathrm{ml}$ of SM buffer on top of plate and shake gently for $15 \mathrm{~min}$ at room temperature.

176 (vi) Collect buffer from top of plate and centrifuge at 4,000 $\mathrm{x}$ g for $5 \mathrm{~min}$.

177 (vii) Collect phage lysate and store at $4{ }^{\circ} \mathrm{C}$ until clean up. Optional: Titer the lysate via plaque assay to ensure initial high titer.

179

180 2B| Phage propagation via liquid lysate

181 (i) Grow E. coli bacterial host in LB broth overnight at $37^{\circ} \mathrm{C}$.

182 (ii) Prepare and autoclave $100 \mathrm{ml}$ of $\mathrm{LB}$ broth supplemented $0.001 \mathrm{M} \mathrm{CaCl}_{2}$ and $\mathrm{MgCl}_{2}$ 183 added in a $250 \mathrm{ml}$ PYREX ${ }^{\circledR}$ screw cap storage bottle and save for Step 3.

184 (iii) Spike LB broth supplemented with $\mathrm{CaCl}_{2}$ and $\mathrm{MgCl}_{2}$ with 0.1 volumes of overnight bacterial host.

Note: For a phage with a high burst size, such as T3, you may need to double volume of host added.

(iv) Incubate with agitation for $1 \mathrm{hr}$ at $37^{\circ} \mathrm{C}$.

190

(v) Add $100 \mu \mathrm{l}$ of high titer phage lysate $\left(>10^{8} \mathrm{PFU} \cdot \mathrm{ml}^{-1}\right)$.

(vi) Incubate at $37^{\circ} \mathrm{C}$ with agitation for $\sim 5 \mathrm{hrs}$ or until lysate clears.

(viii) Collect phage lysate store at $4{ }^{\circ} \mathrm{C}$ until clean up.

193

194

195

196

197

198

199

200

3| Phage cleanup (0.22 $\mu \mathrm{m}$ filtering and chloroform)

(i) Aliquot phage lysate into $50 \mathrm{ml}$ sterile falcon centrifuge tubes and centrifuge at 4,000 x $\mathrm{g}$ for $20 \mathrm{~min}$.

(ii) Carefully collect supernatant using a serological pipette and transfer into properly labeled sterile falcon tube.

(iii) Filter-sterilize the phage supernatant using a $0.22 \mu \mathrm{m}$ filter to yield a bacterial cell-free phage lysate. 
201 (iv) Add 0.1 volumes of chloroform to the supernatant, vortex, and incubate at room temp

202

203

204

205

206

207

208

209

210

211

212

213

214

215

216

217

218

219

220

221

222

223

224

225

226

227

228

229

230 for $10 \mathrm{~min}$.

Note: Lipid enveloped phages are sensitive to chloroform and titer can be significantly reduced. If a drop in titer is observed skip this step.

(v) Centrifuge at $4,000 \mathrm{x}$ g for $5 \mathrm{~min}$ and transfer supernatant into $250 \mathrm{ml}$ PYREX ${ }^{\circledR}$ screw cap storage bottle and store at $4{ }^{\circ} \mathrm{C}$ until concentration.

Optional: Titer the lysate via plaque assay to ensure initial high titer.

4| Phage concentration and wash via ultrafiltration

(i) Add $\sim 15 \mathrm{ml}$ of phage lysate into the upper reservoir of Amicon filter device.

(ii) Centrifuge Amicon at 4,000 $\mathrm{xg}$ for $\sim 5 \mathrm{~min}$.

Note: Centrifugation times will vary based on phage type and titer. It is important not to spin the device dry. If unsure about centrifugation times select a shorter spin time, check the lysate level, and adjust spin times appropriately.

(iii) Carefully discard the filtrate into a waste bucket and add another volume of phage lysate to the sample filter cup and repeat centrifugation.

Note: The same device can be used to concentrate large volumes of phage lysate (>100 ml) achieving an approximate 90\% decrease with minimal loss in phage titer.

(iv) Repeat step until all phage lysate has been concentrate to $<10 \mathrm{ml}$.

(v) Add $\sim 15 \mathrm{ml}$ of SM buffer into the upper reservoir containing concentrate phage lysate and centrifuge at $4,000 \mathrm{x}$ g for $\sim 5 \mathrm{~min}$ to wash phage lysate.

Note: SM buffer was chosen as it is suitable for the long-term storage of T-phage, but any appropriate storage buffer can be used. It is important not to spin the device dry, adjust centrifugation times accordingly.

(vi) Repeat wash step and concentrate washed phage lysate to $<10 \mathrm{ml}$.

(vii) Using a pipette, carefully collect phage lysate from the upper reservoir and gently wash the surface of the upper reservoir.

(viii) Collect $<10 \mathrm{ml}$ of concentrated and purified phage lysate. Titer phage concentrate and record PFU'ml ${ }^{-1}$.

2315 | Endotoxin removal (Morrison \& Leive, 1975; Szermer-Olearnik \& Boratyński, 2015) 
232 Notes:

233 - This method is adapted from Szermer-Olearnik \& Boratyński (2015), which demonstrates

234 the efficient removal of endotoxins from bacteriophage lysates using water immiscible

235 solvents that are subsequently removed via dialysis. For detailed explanation of the

236 methodology please see Morrison \& Leive (1975) and Szermer-Olearnik \& Boratyński

237 (2015).

238

239

240

241

242

243

244

245

246

247

248

249

250

251

252

- Our adapted method uses a speed vacuum to remove residual organic solvent from phage lysates, instead of the lengthy dialysis washes with similar efficiency.

- This step is optional. If you do not require removal of bacterial endotoxins from your phage preparations please go to step 7 .

253

$254 \mathbf{6 A}$ | Dialysis removal of organic solvent (Szermer-Olearnik \& Boratyński, 2015)

255 Notes:

256

257

258

259

260

(i) Add 0.4 volumes of 1-octanol to phage concentrate and shake at room temp for $1 \mathrm{hr}$.

(ii) Incubate phage concentrate at $4{ }^{\circ} \mathrm{C}$ for $1.5 \mathrm{hr}$.

(iii) Centrifuge at $4,000 \mathrm{x}$ for $10 \mathrm{~min}$.

(iv) Using a syringe, pierce the bottom of the tube and collect the aqueous phase (bottom layer) that contains your phage, and transfer to a sterile $50 \mathrm{ml}$ falcon tube. Do not collect the organic phage (top layer) or interface as this contains endotoxins.

Note: It is best to leave a small residual amount of phage concentrate behind to reduce the transfer of contaminating endotoxins.

Note: If endotoxin removal is not sufficient the method can be repeated to further reduce endotoxins.

- This method is adapted from Szermer-Olearnik \& Boratyński (2015) and describes the removal of residual organic solvents from phage lysates by dialysis.

- Residual organic solvents disable downstream Pierce ${ }^{\mathrm{TM}}$ LAL Chromogenic Endotoxin Quantitation assays and must be removed in order to accurately quantify endotoxin concentrations.

262

- Due to the ionic concentration of phage SM buffer used you may end up with greater than the starting volume. 
(i) Pre-wet Spectra-Por ${ }^{\circledR}$ dialysis membrane with sterilized $\mathrm{dH}_{2} \mathrm{O}$ according to manufacturers instructions, being careful not to contaminate the inside of the tubing.

(ii) Load maximum of $10 \mathrm{ml}$ of phage concentrate inside of dialysis tubing and seal tightly.

(iii) Dialyze phage concentrate against 2 liters of $25 \%(\mathrm{v} / \mathrm{v})$ ethanol at $4{ }^{\circ} \mathrm{C}$ on a stirring plate for $24 \mathrm{hrs}$ to remove residual 1-butanol, replacing the $25 \%$ ethanol solution four times at $15 \mathrm{hr}, 18 \mathrm{hrs} 21 \mathrm{hrs}$, and $24 \mathrm{hrs}$.

270

(iv) Dialyze phage concentrate against 2 liters of $0.15 \mathrm{M} \mathrm{NaCl}$ solution at $4{ }^{\circ} \mathrm{C}$ on a stirring

272 plate $24 \mathrm{hrs}$ to remove residual ethanol, replacing the $0.15 \mathrm{M} \mathrm{NaCl}$ solution three times after $15 \mathrm{hrs}, 19 \mathrm{hrs}$, and $24 \mathrm{hrs}$.

(vi) Carefully collect phage concentrate by washing the inside of the dialysis tubing. Store concentrate at store $4{ }^{\circ} \mathrm{C}$ until phage bank preparation.

Optional: Titer the lysate via plate lysates to ensure high titer. Perform the Pierce ${ }^{\mathrm{TM}} L A L$ Chromogenic Endotoxin Quantitation according to manufacturers instructions to obtain quantitative endotoxin levels.

279

280

6B| Speed vacuum removal of organic solvent

281

282

Notes:

283

284

285

286

287

288

289

290

291

- This method is a faster alternative to the dialysis method for the removal of residual organic solvents from phage concentrates.

292

(i) Aliquot $\sim 1 \mathrm{~mL}$ of the phage concentrate equally into microcentrifuge tubes.

(ii) Place tubes into a speed vacuum $4{ }^{\circ} \mathrm{C}$, open lids, and centrifuge at $4,000 \mathrm{x}$ g for $3 \mathrm{hrs}$.

(iii) After speed vacuum, phage concentrate volume should be reduced by approximately $30 \%$ and residual 1-octanol evaporated.

(iv) Collect phage concentrate and store $4{ }^{\circ} \mathrm{C}$ until phage bank preparation.

Optional: Titer the lysate via plaque assay to ensure high titer. Perform the Pierce ${ }^{\mathrm{TM}}$ LAL Chromogenic Endotoxin Quantitation according to manufacturers instructions to obtain quantitative endotoxin levels from phage concentrates. 
294 7| Phage bank storage

295 (i) Dilute phage lysate in SM buffer to generate a high-titer working stock.

$296 \quad$ Note: This step largely depends on desired concentration and volume of the phage bank.

297 If you require higher titer phage stocks then dilute less or omit dilution, if greater volume $298 \quad$ is desired then dilute more.

299 (ii) Titer diluted phage concentrate and record PFU. $\mathrm{ml}^{-1}$.

300 (iii) Aliquot phage working stocks into labeled cryo tubes and store at $4{ }^{\circ} \mathrm{C}$.

301

302 - You now have a bank of homogenous, high titer (up to $10^{10-11} \mathrm{PFU} \cdot \mathrm{ml}^{-1}$ ) phage bank for 303 laboratory testing.

304 - From our experience the use of homogenous and standardized phage banks can 305

306 significantly reduce the experimental variability for the molecular characterization of

307

308 


\section{Results}

310 Phage purification and concentration. Here we used T4 phage as a representative phage for our

311 Phage On Tap (PoT) phage purification protocol (Figure 1). We tested the speed and efficiency

312 of the PoT purification protocol (Figure 2A) compared against the traditional method (Figure

313 2B), which involved phage propagation, centrifugation, filtering and purification via cesium

314 chloride ultracentrifugation and dialysis (Fig. S1 and S2) (Adams, 1959). Both methods started

315 with $110 \mathrm{~mL}$ of total lysate and comparable titers. The PoT protocol consisted of phage clean up,

316 concentration and washing via ultrafiltration and resulted in a $93 \%$ reduction in volume with a

317 phage recovery efficiency of $85 \%$, while taking just two days to complete. Comparatively, the

318 traditional method, which consisted of phage clean up, cesium chloride ultracentrifugation and

319 dialysis, resulted in an $80 \%$ reduction in volume, with a $4 \%$ phage recovery efficiency and taking

320 five days to complete.

321

322 Endotoxin content. Phage preparations are often contaminated with bacterial endotoxins and their 323 removal from phage preparations is required for some applications (Merril, Scholl \& Adhya, 324 2003; Chan, Abedon \& Loc-Carrillo, 2013). Endotoxin concentrations of T4 lysates from the 325 PoT protocol and traditional method were measured using the LAL chromogenic endotoxin 326 quantitation kits to compare the efficiency of endotoxin removal (Figure 3). The endotoxin 327 content of the raw lysates were $4 \times 10^{4} \mathrm{EU} \cdot \mathrm{ml}^{-1}$, but post clean up neither the PoT nor the 328 traditional method achieved sufficient reductions in endotoxin levels $\left(<20 \mathrm{EU} \cdot \mathrm{ml}^{-1}\right)$, although the

329 PoT protocol did perform marginally better, with a reduction to $8 \times 10^{3}$ compared to $3 \times 10^{4} \mathrm{EU} \cdot \mathrm{ml}^{-}$

$330{ }^{1}$ for the traditional method, respectively.

331

332 Endotoxin removal. In order to generate phage banks with endotoxin concentrations of $<20$ $333 \mathrm{EU} \cdot \mathrm{ml}^{-1}$, we adapted a recently published protocol detailing the removal of endotoxins through 334 extraction with organic solvents and subsequent dialysis washes (Morrison \& Leive, 1975;

335 Szermer-Olearnik \& Boratyński, 2015). The T4 phage concentrate from our PoT protocol was 336 processed through the endotoxin removal protocol using the organic solvent 1-Octanol (Figure 337 4A). After endotoxin removal there was a slight reduction in phage volume and titer, likely due

338 to loss of phage concentrate that was left near the organic layer. Unfortunately endotoxin 339 quantification at this step was not possible due to residual 1-Octanol in the phage concentrate 
340 disabling LAL endotoxin test (Szermer-Olearnik \& Boratyński, 2015). In order to remove

341 residual 1-Octanol, phage concentrates were split evenly, processed through dialysis and speed

342 vacuum ( $5 \mathrm{~mL}$ each) and endotoxin levels quantified (Figure 4B). Following dialysis washes,

343 we were capable of producing a T4 phage concentrate with $14 \mathrm{EU} \cdot \mathrm{ml}^{-1}$, with a $30 \%$ increase in

344 volume (likely due to water movement to the higher ionic strength SM buffer), and a phage

345 recovery efficiency of $60 \%$ within four days. The major limitation with this method is the

346 lengthy dialysis step required to remove residual 1-Octanol solvents from the phage concentrate,

347 taking upwards of $48 \mathrm{hrs}$ to complete. We tested a faster speed vacuum method to remove this

348 residual solvent, taking just $3 \mathrm{hrs}$ to complete and producing a lysate with a slightly increased

349 endotoxin content of $27 \mathrm{EU} \cdot \mathrm{ml}^{-1}$, with a $20 \%$ reduction in volume and a phage recovery

350 efficiency of $47 \%$.

352 Phage banks and storage: Phage banks were produced from purified concentrates to provide

353 homogenous, high titer, endotoxin reduced phage stock for repeat molecular testing and

354 characterization. As such it was important to ensure the stability of phage banks under repeated

355 laboratory use. We tested a range of conditions for the shot-term storage and repeated use of T4

356 phage banks, including $4{ }^{\circ} \mathrm{C}$ in SM buffer, liquid nitrogen in $50 \% \mathrm{v} / \mathrm{v}$ glycerol, liquid nitrogen in

$3575 \% \mathrm{v} / \mathrm{v}$ DMSO, $-20{ }^{\circ} \mathrm{C}$ in $50 \% \mathrm{v} / \mathrm{v}$ glycerol and $-80{ }^{\circ} \mathrm{C}$ in $50 \% \mathrm{v} / \mathrm{v}$ glycerol (Figure 5A). Phage

358 bank storage at $4{ }^{\circ} \mathrm{C}$ in SM buffer showed minimal reduction in titer for short-term storage of

359 high-use phage banks. Freezing of high-use phage banks is not recommended, as the repeated

360 freeze-thaw cycles from the frozen preparations likely damaged phage, reducing titer. If long-

361 term storage of phage banks is required, we recommend storage in liquid nitrogen with $5 \% \mathrm{v} / \mathrm{v}$

362 DMSO with minimal freeze-thaws, as this showed lowest loss of phage titer over a 3-month

363 period. However, prior to use of frozen phage banks, it is advised to wash phage lysate with SM

364 buffer through an ultrafiltration unit to remove any residual DMSO. Finally, T4 phage

365 morphology and structure following PoT protocol was determined by transmission electron

366 microscopy (TEM) (Figure 5B). T4 phage showed tails that were not contracted and intact

367 capsid structures, indicating that PoT protocol did not negatively impact phage structure and 368 viability. 
370 Applicability of Phage on Tap protocol: As the PoT protocol is based on an ultrafiltration

371 methodology, which we believe is broadly applicable to other tailed phages that can be isolate

372 and cultured to high titer. To test this, we processed four tailed phages, including T4 $\Delta h o c-\mathrm{a}$

373 Myoviridae infecting E. coli; T3 - a Podoviridae infecting E. coli; T5 - a Siphoviridae infecting

374 E. coli; and Spp1 - a Siphoviridae infecting the Gram-positive Bacillus subtilis, through the PoT

375 protocol (Figure 6). Phages T3, T5 and Spp1 were propagated by plate lysates, while T4 $4 h o c$

376 phage was propagated by liquid lysate. All phages were processed through to a concentrate with

377 a $>50 \%$ phage recovery efficiency, followed by 1-Octanol endotoxin removal and processing by

378 dialysis and speed vacuum. All phages showed a large reduction in endotoxin concentrations, but

379 none were below the desired $<20 \mathrm{EU} \cdot \mathrm{ml}^{-1}$ with the exception of Spp1 phage, which infects the

380 Gram-positive Bacillus subtilis bacterial host. Spp1 phage lysate produced an already low

381 endotoxin lysate that we further reduced to $<1 \mathrm{EU} \cdot \mathrm{ml}^{-1}$. The dialysis method was more efficient

382 than speed vacuum at removing bacterial endotoxins, but took $48 \mathrm{hrs}$ to complete.

383 Comparatively, the speed vacuum method took only $3 \mathrm{hrs}$ to complete, producing a phage

384 concentrate with an average 2-fold higher endotoxin concentration than the dialysis method with

385 a comparable titer. Overall, all of the phages processed through the PoT protocol generated high

386 titer, homogenous phage banks with a significantly reduced in endotoxin levels. 


\section{Discussion}

389 Traditional methods for the isolation and generation of phages lysates often involve

390 centrifugation, precipitation with Polyethylene Glycol (PEG), ultracentrifugation in cesium

391 chloride $(\mathrm{CsCl})$ gradients, followed by dialysis and storage. These procedures are lengthy and

392 time consuming and generate phage lysates with variable titer, endotoxin, and ionic

393 concentration. Here we present a fast and efficient method to produce homogeneous phage banks

394 for laboratory testing and molecular characterization. Our method focused on T4 phage, but is

395 broadly applicable to other phages that can be isolated in high titer $\left(>10^{9} \mathrm{PFU} \cdot \mathrm{ml}^{-1}\right)$. Specifically,

396 the T4 phage was propagated with E. coli bacterial host in liquid lysate, purified by 397 centrifugation, $0.22 \mu \mathrm{m}$ filtration and chloroform treatment, concentrated by ultrafiltration

398 centrifugation, and stored in large phage banks at $4{ }^{\circ} \mathrm{C}$ in buffer. Unfortunately, neither the PoT 399 protocol nor the traditional methods were effective at significantly reducing bacterial endotoxins 400 from phage preparations, and additional purification steps were required.

401

402 Numerous methods and commercially available kits are available for the removal of bacterial 403 endotoxins (Merril et al., 1996; Boratyński et al., 2004; Merabishvili et al., 2009; Oślizło et al., 404 2011; Branston, Wright \& Keshavarz-Moore, 2015), yet many of these are either highly specific, 405 time consuming, laborious, or expensive. Szermer-Olearnik \& Boratyński recently proposed the 406 use of an organic solvent to successfully reduce bacterial endotoxin from phage lysates $(<20$ $407 \mathrm{EU} \cdot \mathrm{ml}^{-1}$ ). The method is cheap, broadly applicable, and capable of endotoxin removal regardless 408 of initial variations in titer and endotoxin levels, but does rely on multiple dialysis steps that are 409 time consuming (Morrison \& Leive, 1975; Szermer-Olearnik \& Boratyński, 2015). It may be 410 possible to shorten the dialysis method through more frequent changes in buffer solution, 411 although this needs to be confirmed. Here we modify this method by replacing the long dialysis 412 steps with a speed vacuum step for the removal of residual 1-octanol from the lysates. The speed 413 vacuum modification was not as efficient as the dialysis step for the removal of bacterial 414 endotoxins, but was significantly shorter to complete - taking just $3 \mathrm{hrs}$ compared to the $48 \mathrm{hrs}$ 415 required for dialysis. Using this modification we are able to produce homogenous, high-titer, 416 endotoxin-reduced phage banks within two days. 
418 Phage are generally quite stable at high concentrations when stored at $4{ }^{\circ} \mathrm{C}$, free of bacterial

419 debris, protected from light exposure, and in appropriately buffered solution with a pH between

420 5-9 (Adams, 1959; Clark, 1962; Wommack et al., 1996; Jończyk et al., 2011a). Although most

421 tailed and filamentous phages can be easily stored under these conditions for 5-10+ years, it is

422 always best to determine the optimal storage conditions for each phage of interest. Phage are

423 generally sensitive to freezing, thawing, and lyophilization and titer is known to vary with

424 storage time (Clark, 1962; Clark \& Klein, 1966; Clark \& Geary, 1973; Jończyk et al., 2011b).

425 After monitoring the degradation of numerous phages for over 21 years, it was shown that phage

426 storage at $4{ }^{\circ} \mathrm{C}$ and $-80{ }^{\circ} \mathrm{C}$ was suboptimal compared to storage in liquid nitrogen $\left(-196{ }^{\circ} \mathrm{C}\right)$

427 (Ackermann, Tremblay \& Moineau, 2003). However, due to their diversity and the difficulties

428 associated with accurate monitoring, the optimal conditions for long-term storage of phage ( $>10$ 429 years) remains uncertain. The purpose of PoT protocol was to generate homogenous, high-titer

430 phage banks for regular and consistent laboratory use. For this purpose the storage of phage in

$431 \mathrm{SM}$ buffer at $4{ }^{\circ} \mathrm{C}$ over three months showed no noticeable depreciation in titer, which is 432 consistent with previously described short-term storage conditions.

433

434 The Phage On Tap (PoT) protocol described here takes two days to produce high titer, 435 homogenous, endotoxin reduced phage banks for molecular characterizations. In comparison, the 436 traditional method can take upwards of five days to complete, is laborious, and generates a lower 437 titer phage lysate with high endotoxin levels. Traditional phage propagations suffer from further 438 variability in titer, salts, and bacterial contaminants between successive lysates. Eliminating this 439 variability through the use of PoT phage banks has been critical for the accurate analysis and 440 molecular investigations of phage within the context of eukaryotic hosts (Barr et al., 2013, 2015). 441 Phage banks can be easily stored at $4^{\circ} \mathrm{C}$ in SM buffer while maintaining their viability under 442 continuous high-use. The PoT protocol is efficient, can be easily completed in the laboratory, 443 may be among the least costly, and generates large homogenous phage banks for repeated use.

\section{Acknowledgements}

446 We thank the San Diego State University TEM Facility for the help with the TEM 447 analyses. 


\section{Figures}

452

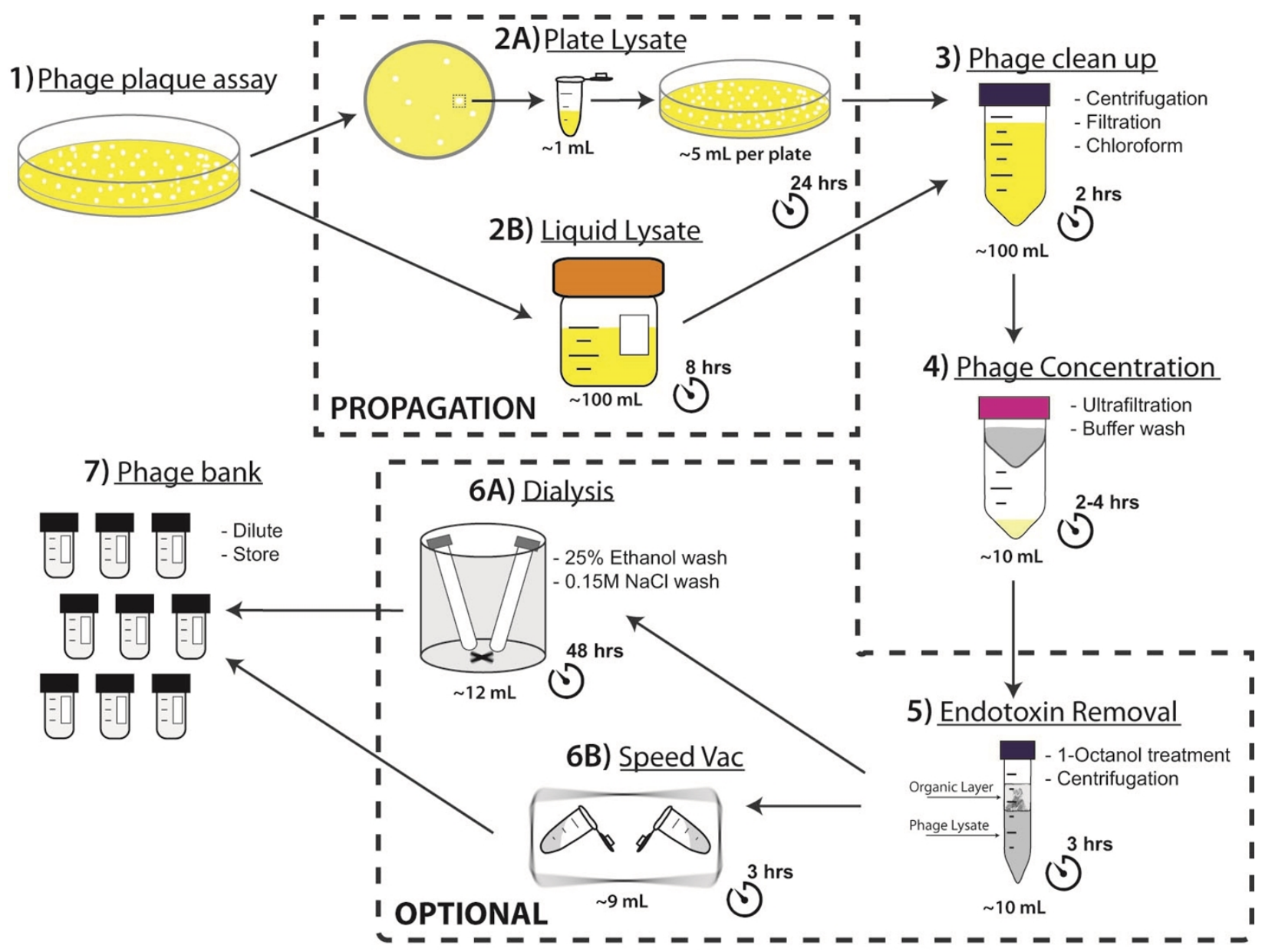

454 Figure 1. Flow chart of the Phage on Tap (PoT) protocol for the production of high titer,

455 homogenous, endotoxin reduced phage banks within two days. Phage titers (PFU. $\mathrm{ml}^{-1}$ ) and 456 volumes $(\mathrm{mL})$ were recorded after each step. Endotoxin removal is adapted from Szermer457 Olearnik \& Boratyński (2015). 
A) Phage on Tap (PoT)

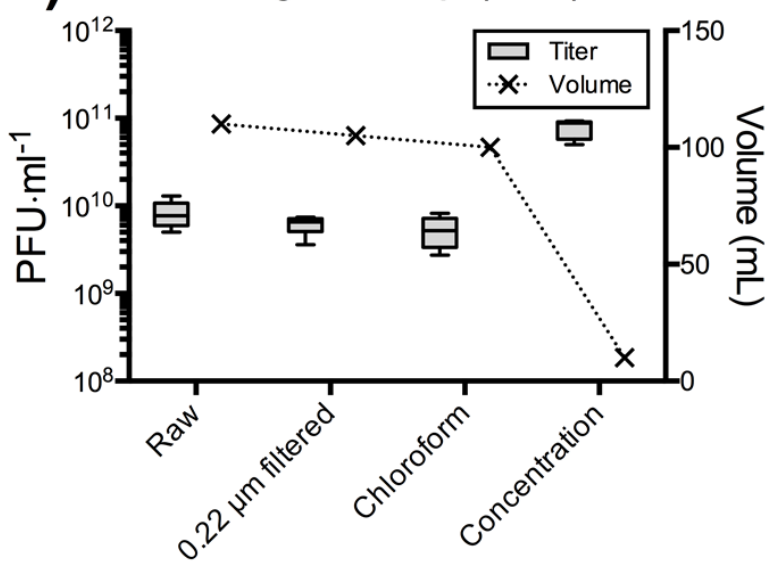

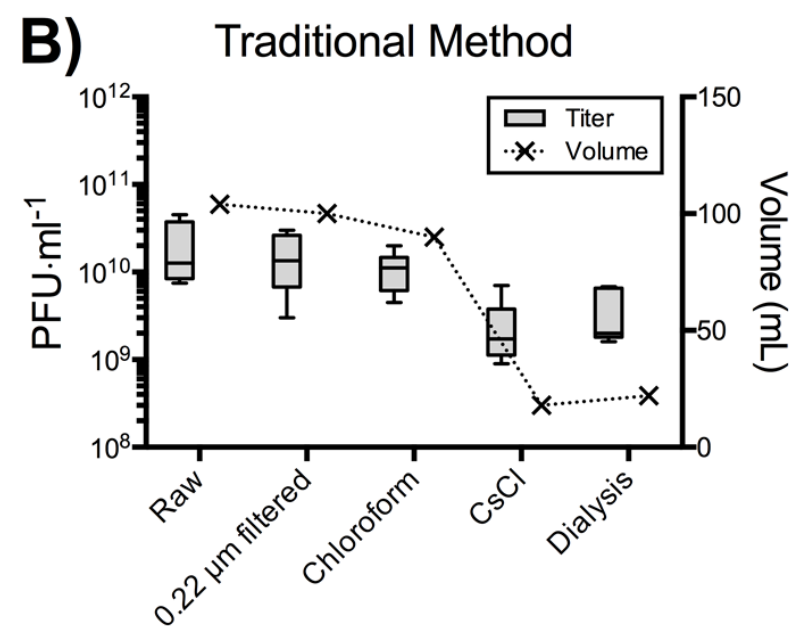

459 Figure 2. A comparison between the Phage on Tap (PoT) protocol and traditional method for the

460 purification and concentration of laboratory phage stocks. (A) The PoT protocol generated a 10 $461 \mathrm{~mL}$ phage stock $\left(7.7 \times 10^{10} \mathrm{PFU} \cdot \mathrm{ml}^{-1}\right)$ with a phage recovery efficiency of $85 \%$ within two days. 462 (B) The traditional method generated a $22 \mathrm{~mL}$ phage lysate $\left(3.7 \times 10^{9} \mathrm{PFU} \cdot \mathrm{ml}^{-1}\right)$ with a phage 463 recovery efficiency of $4 \%$ in five days.

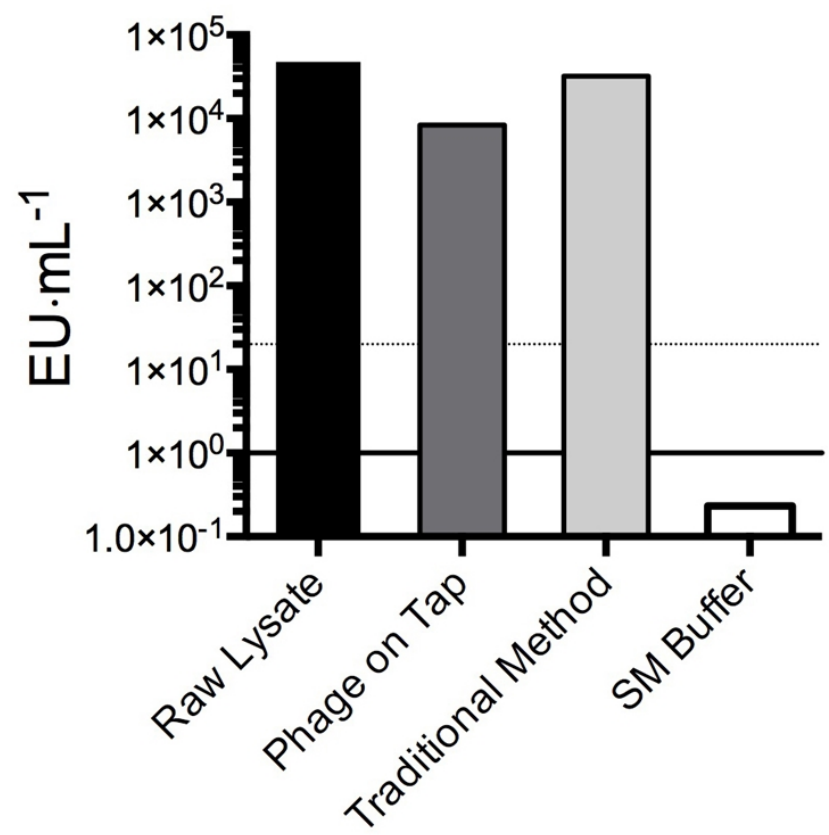

465 Figure 3. Endotoxin concentration $\left(\mathrm{EU} \cdot \mathrm{ml}^{-1}\right)$ of phage preparations as measured by the Pierce ${ }^{\mathrm{TM}}$ 466 LAL Chromogenic Endotoxin Quantitation Kit. Raw lysates had starting endotoxin 467 concentration of $4.7 \times 10^{4} \mathrm{EU} \cdot \mathrm{ml}^{-1}$. The PoT protocol and traditional method reduced endotoxin 468 concentrations to $8.3 \times 10^{3}$ and $3.2 \times 10^{4} \mathrm{EU} \cdot \mathrm{ml}^{-1}$, respectively. Filter-sterilized SM buffer $(0.2$ 
$469 \mathrm{EU}^{\mathrm{m}} \mathrm{ml}^{-1}$ ) is shown as a negative control, dotted line represent the desired $20 \mathrm{EU} \cdot \mathrm{ml}^{-1}$ cut off. 470 Endotoxin concentration data shown are averages of triplicates from a single experiment.

471

472

473

474

475

476

477

478

479

480
A)

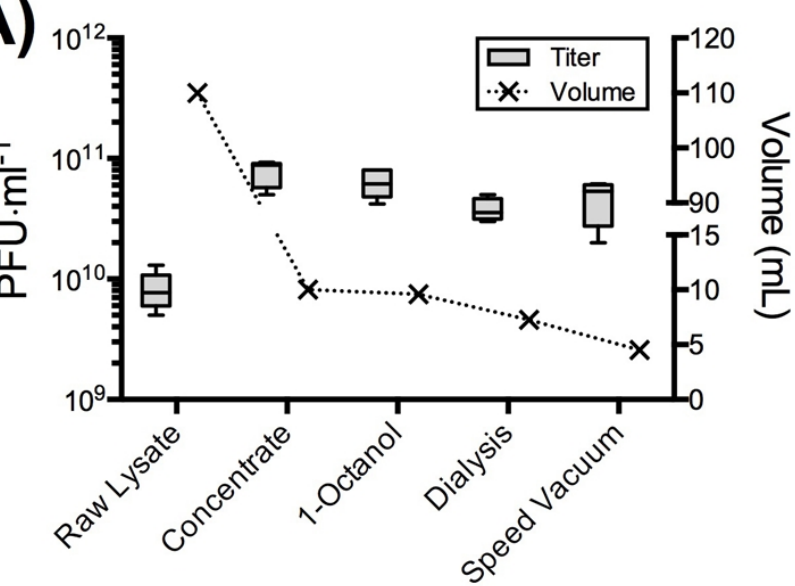

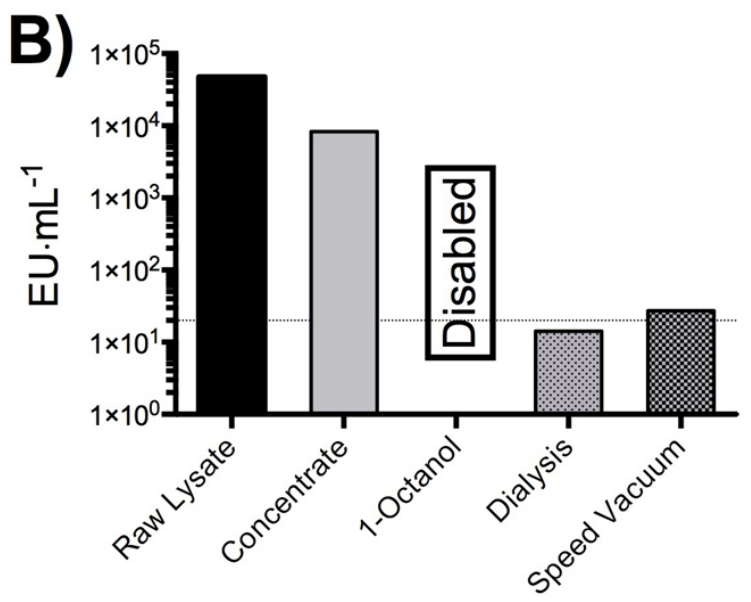

Figure 4. Phage on Tap (PoT) protocol processed for the removal of bacterial endotoxins by 1Octanol and solvent removal by dialysis and speed vacuum. (A) The PoT protocol processed for endotoxin removal by the dialysis and speed vacuum methods generated a $7.2 \mathrm{~mL}\left(3.8 \times 10^{10}\right.$ $\left.\mathrm{PFU} \cdot \mathrm{ml}^{-1}\right)$ and $4.2 \mathrm{~mL}\left(4.7 \times 10^{10} \mathrm{PFU} \cdot \mathrm{ml}^{-1}\right)$ phage banks, respectively. (B) Endotoxin concentrations of dialysis $\left(14 \mathrm{EU} \cdot \mathrm{ml}^{-1}\right)$ and speed vacuum $\left(27 \mathrm{EU} \cdot \mathrm{ml}^{-1}\right)$ processed PoT phage banks. Quantification of 1-Octanol treated concentrate was not possible due to residual solvent disabling the LAL quantification test, dotted line represent the desired $20 \mathrm{EU} \cdot \mathrm{ml}^{-1}$ cut off. 
481
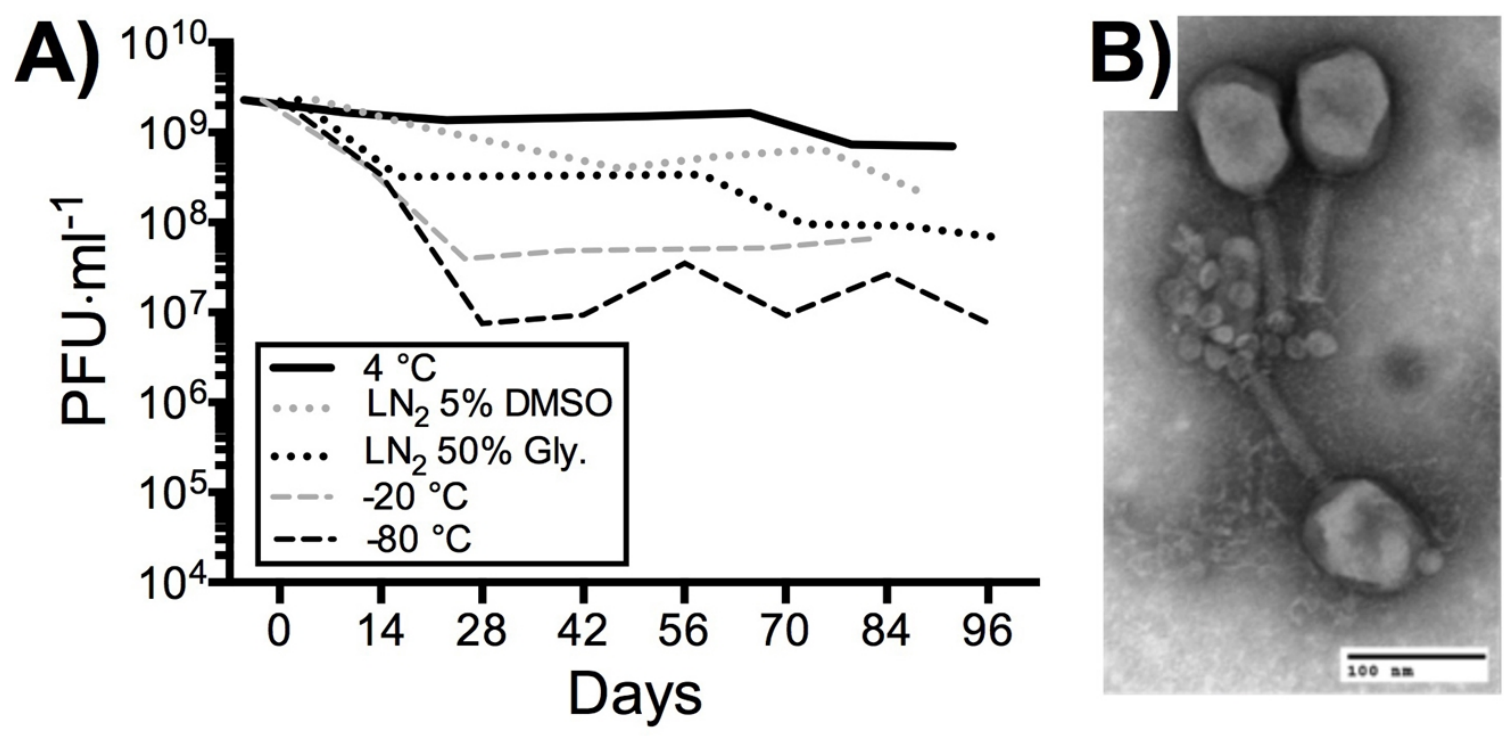

Figure 5. Stability of T4 phage processed through PoT under high-use laboratory conditions. (A) 484 The mean titer of T4 phage stored at $4{ }^{\circ} \mathrm{C}$ in $\mathrm{SM}$ buffer, liquid nitrogen $\left(\mathrm{LN}_{2}\right)$ in $5 \%(\mathrm{v} / \mathrm{v})$ 485 DMSO, $\mathrm{LN}_{2}$ in $50 \%$ (v/v) glycerol, $-20{ }^{\circ} \mathrm{C}$ in $50 \%$ glycerol and $-80{ }^{\circ} \mathrm{C}$ in $50 \%$ glycerol. Phage 486 stocks were tittered in duplicate under high-use conditions (full titers Fig. S4). (B) Negative stain 487 transmission electron micrograph of T4 phage processed through the PoT protocol (scale bar = $488100 \mathrm{~nm})$. 

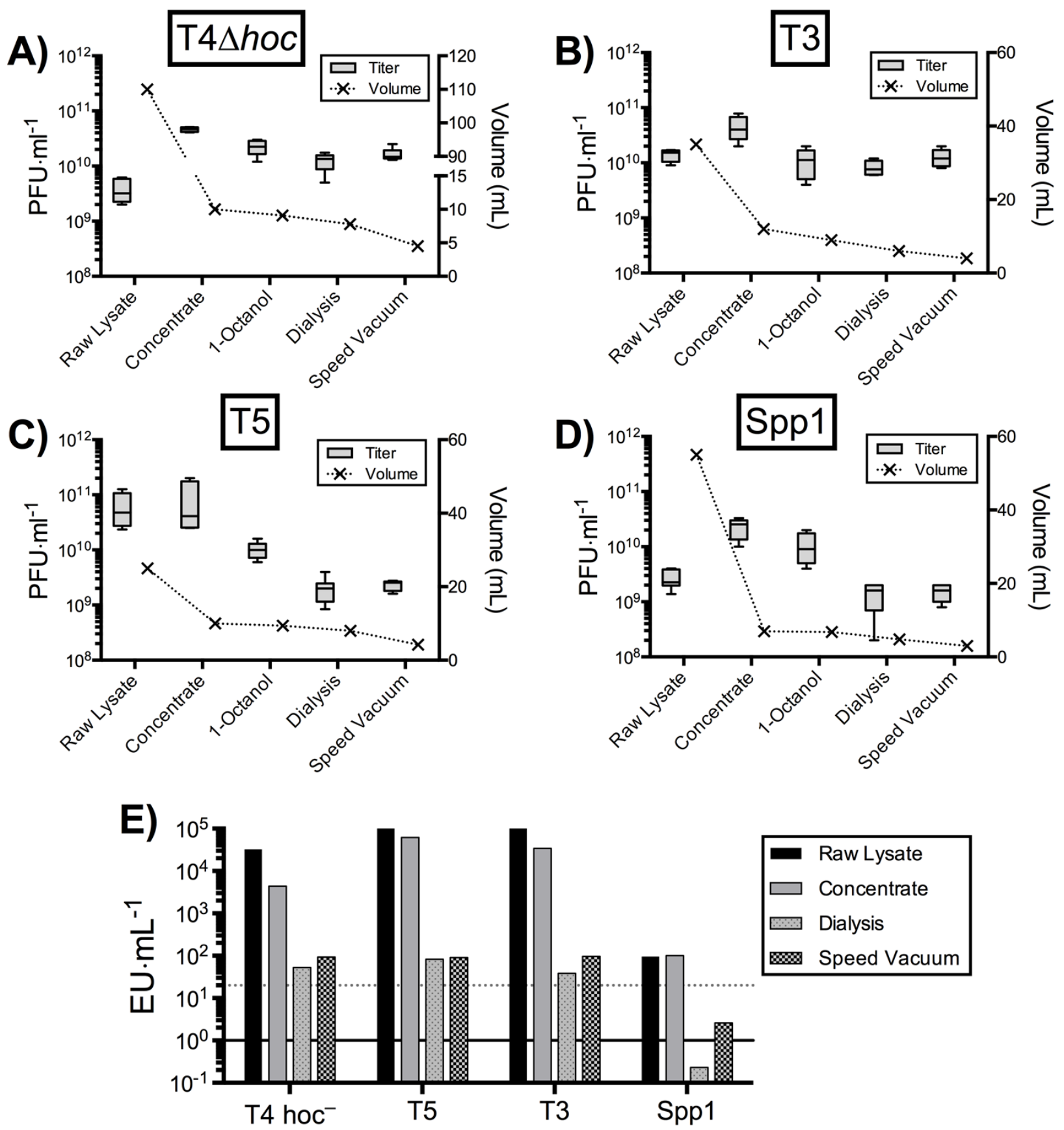

491 Figure 6. Applicability of Phage on Tap (PoT) protocol for the generation of high-titer,

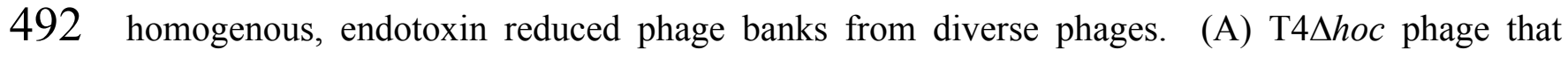
493 infects $E$. coli bacterial host propagated by liquid lysate $\left(2.2 \times 10^{10} \mathrm{PFU} \cdot \mathrm{ml}^{-1}\right)$. (B) T3 phage that 494 infects E. coli bacterial host propagated by plate lysate $\left(1.1 \times 10^{10} \mathrm{PFU} \cdot \mathrm{ml}^{-1}\right)$. (C) T5 phage that 495 infects $E$. coli bacterial host propagated by plate lysate $\left(2 \times 10^{9} \mathrm{PFU}^{\mathrm{m}} \mathrm{ml}^{-1}\right)$. (D) Spp1 phage that 496 infects B. subtilis Gram-positive bacterial host propagated by plate lysate $\left(1.5 \times 10^{9} \mathrm{PFU} \cdot \mathrm{ml}^{-1}\right)$. 497 (E) Endotoxin concentrations of raw lysates, PoT concentrate, dialysis and speed vacuum treated 
498 phage banks, dotted line represent the desired $20 \mathrm{EU} \cdot \mathrm{ml}^{-1}$ cut off. Overall, a decrease in volume 499 (ml) of the lysates was observed after each step of the procedure.

500

501 


\section{References}

503 Abedon ST., Kuhl SJ., Blasdel BG., Kutter EM. 2011. Phage treatment of human infections.

504

505

506

507

508

509

510

511

512

513

514

515

516

517

518

519

520

521

522

523

524

525

526

527 Bacteriophage 1:66-85.

Ackermann H., Tremblay D., Moineau S. 2003. Long-Term Bacteriophage Preservation. :2-7. Adams MH. 1959. Bacteriophages. New York, N. Y.: Interscience.

Alexander C., Rietschel ET. 2001. Invited review: Bacterial lipopolysaccharides and innate immunity. Journal of Endotoxin Research 7:167-202.

Barr JJ., Auro R., Furlan M., Whiteson KL., Erb ML., Pogliano J., Stotland A., Wolkowicz R., Cutting AS., Doran KS., Salamon P., Youle M., Rohwer F. 2013. Bacteriophage adhering to mucus provide a non-host-derived immunity. Proceedings of the National Academy of Sciences 110:10771-10776.

Barr JJ., Auro R., Sam-Soon N., Kassegne S., Peters G., Bonilla N., Hatay M., Mourtada S., Bailey B., Youle M., Felts B., Baljon A., Nulton J., Salamon P., Rohwer F. 2015. Subdiffusive motion of bacteriophage in mucosal surfaces increases the frequency of bacterial encounters. Proceedings of the National Academy of Sciences 112:201508355.

Batista P de OMD., Lopes AM., Mazzola PG., Yagui C de OR., Penna TCV., Pessoa Júnior A. 2013. Methods of endotoxin removal from biological preparations : a review. Journal of Pharmacy \& Pharaceutical Sciences 10:388-404.

Boratyński J., Syper D., Weber-Dabrowska B., Łusiak-Szelachowska M., Poźniak G., Górski A. 2004. Preparation of endotoxin-free bacteriophages. Cellular \& molecular biology letters 9:253-9.

Bourdin G., Schmitt B., Marvin Guy L., Germond J-E., Zuber S., Michot L., Reuteler G., Brüssow H. 2014. Amplification and purification of T4-like Escherichia coli phages for phage therapy: from laboratory to pilot scale. Applied and environmental microbiology 80:1469-76.

Branston SD., Wright J., Keshavarz-Moore E. 2015. A non-chromatographic method for the removal of endotoxins from bacteriophages. Biotechnology and bioengineering.

Bruttin A., Brüssow H. 2005. Human volunteers receiving Escherichia coli phage T4 orally: a safety test of phage therapy. Antimicrobial agents and chemotherapy 49:2874-8.

Carlson K. 2004. Working with bacteriophages: common techniques and methodological approaches. CRC Press.

Chan BK., Abedon ST., Loc-Carrillo C. 2013. Phage cocktails and the future of phage therapy. Future microbiology 8:769-83.

Clark WA. 1962. Comparison of Several Methods for Preserving Bacteriophages. Applied Environmental Microbiology 10:466-471.

Clark WA., Geary D. 1973. Preservation of bacteriophages by freezing and freeze-drying. Cryobiology 10:351-360.

Clark WA., Klein A. 1966. The stability of bacteriophages in long term storage at liquid nitrogen temperatures. Cryobiology 3:68-75.

Erickson HP. 2009. Size and shape of protein molecules at the nanometer level determined by sedimentation, gel filtration, and electron microscopy. Biological procedures online 11:3251.

Gorbet MB., Sefton M V. 2005. Endotoxin: the uninvited guest. Biomaterials 26:6811-7.

Jończyk E., Kłak M., Międzybrodzki R., Górski A. 2011. The influence of external factors on bacteriophages--review. Folia microbiologica 56:191-200.

Merabishvili M., Pirnay J-P., Verbeken G., Chanishvili N., Tediashvili M., Lashkhi N., Glonti 
548

549

550

551

552

553

554

555

556

557

558

559

560

561

562

563

564

565

566

567

568

569

570

571

572

573

574

575

576

577

578

579

580

581

582

583

584

T., Krylov V., Mast J., Van Parys L., Lavigne R., Volckaert G., Mattheus W., Verween G., De Corte P., Rose T., Jennes S., Zizi M., De Vos D., Vaneechoutte M. 2009. Qualitycontrolled small-scale production of a well-defined bacteriophage cocktail for use in human clinical trials. PloS one 4:e4944.

Merril CR., Biswas B., Carlton R., Jensen NC., Creed GJ., Zullo S., Adhya S. 1996. Longcirculating bacteriophage as antibacterial agents. Proceedings of the National Academy of Sciences 93:3188-3192.

Merril CR., Scholl D., Adhya SL. 2003. The prospect for bacteriophage therapy in Western medicine. Nature reviews. Drug discovery 2:489-97.

Morrison DC., Leive L. 1975. Fractions of lipopolysaccharide from Escherichia coli O111:B4 prepared by two extraction procedures. Journal of Biological Chemistry 250:2911-2919.

Morrison DC., Ulevitch RJ. 1978. The effects of bacterial endotoxins on host mediation systems. A review. The American journal of pathology 93:526-618.

Oślizło A., Miernikiewicz P., Piotrowicz A., Owczarek B., Kopciuch A., Figura G., Dąbrowska K. 2011. Purification of phage display-modified bacteriophage T4 by affinity chromatography. BMC biotechnology 11:59.

Pabst MJ., Pabst KM., Handsman DB., Beranova-Giorgianni S., Giorgianni F. 2008. Proteome of monocyte priming by lipopolysaccharide, including changes in interleukin-1beta and leukocyte elastase inhibitor. Proteome science 6:13.

Raetz CRH., Reynolds CM., Trent MS., Bishop RE. 2007. Lipid A modification systems in gram-negative bacteria. Annual review of biochemistry 76:295-329.

Rietschel E., Kirikae T., Schade F., Mamat U., Schmidt G., Loppnow H., Ulmer A., Zahringer U., Seydel U., Di Padova F. 1994. Bacterial endotoxin: molecular relationships of structure to activity and function. FASEB J 8:217-225.

Seeley ND., Primrose SB. 1982. A Review: The isolation of bacteriophages from the environment. Journal of Applied Bacteriology 53:1-17.

Suttle CA., Chan AM., Cottrell MT. 1991. Use of Ultrafiltration To Isolate Viruses from Seawater Which Are Pathogens of Marine Phytoplankton. Applied Environmental Microbiology 57:721-726.

Szermer-Olearnik B., Boratyński J. 2015. Removal of endotoxins from bacteriophage preparations by extraction with organic solvents. PloS one 10:e122672.

Wommack K., Hill R., Muller T., Colwell R. 1996. Effects of sunlight on bacteriophage viability and structure. Applied Environmental Microbiology 62:1336-1341.

Yamamoto KR., Alberts BM., Benzinger R., Lawhorne L., Treiber G. 1970. Rapid bacteriophage sedimentation in the presence of polyethylene glycol and its application to large-scale virus purification. Virology 40:734-744. 